\title{
A Novel Rotor Profile Error Tracing and Compensation Strategy for High Precision Machining of Screw Rotor Based on Trial Cutting of Limited Samples
}

\author{
ZhiHuang Shen, ${ }^{1}$ Bin Yao, ${ }^{1}$ BinQiang Chen, ${ }^{2}$ Wei Feng, ${ }^{1}$ and XiangLei Zhang ${ }^{1}$ \\ ${ }^{1}$ Department of Mechanical and Electrical Engineering, School of Physics and Mechanical \& Electrical Engineering, \\ Xiamen University, Xiamen 361005, China \\ ${ }^{2}$ State Key Laboratory for Manufacturing and Systems Engineering, School of Mechanical Engineering, \\ Xian Jiaotong University, Xian 710049, China
}

Correspondence should be addressed to Bin Yao; yaobin@xmu.edu.cn

Received 23 July 2014; Accepted 14 November 2014

Academic Editor: Yaguo Lei

Copyright (C) 2015 ZhiHuang Shen et al. This is an open access article distributed under the Creative Commons Attribution License, which permits unrestricted use, distribution, and reproduction in any medium, provided the original work is properly cited.

\begin{abstract}
The machining precision plays an important role in the operation reliability and service life of screw rotors. However, the actual machined rotor profile of screw rotors is different from its theoretical profile due to the errors of the machine tool. This paper proposes a novel method of error tracing and compensation to reduce the machined errors of rotor profile on the basis of the limited sample trials, and the method is based on a matrix of error compensation. The errors of rotor profile are obtained based on limited sample trials machining of screw rotors,and fitted into piecewise smooth data. A matrix of error compensation is established to compute the errors of rotor profile to generate a compensated rotor profile. The compensated rotor profile is then used to regenerate forming tool and a new rotor product is processed on the same machine tool. And the errors of new rotor profile are smaller and can be reduced within $(-0.01 \mathrm{~mm}, 0.01 \mathrm{~mm})$ after compensations. Finally, the actual machining examples illustrate that the method of error compensation can not only satisfy the machining requirement of high-precision rotors, but also has the characteristics of good stability and applicability.
\end{abstract}

\section{Introduction}

The advantages of twin-screw compressor include high reliability, good adaptability, ease of operation and maintenance, and the ability to transport various mixed liquids. Therefore, the twin-screw compressor has been widely used in automotive, compression, refrigeration, metallurgy, buildings, and other industrial fields. The design, manufacturing, and operational maintenance of screw rotor have been a topic that attracts more and more attention. As for bearings [1], gears [2], and shafts [3-5], their operation conditions can be investigated by many advanced monitoring technologies. The screw rotor is a kind of nonstandard part whose manufacturing quality is not so easy to guarantee. And a screw rotor of inferior quality has a negative effect on the fault diagnosis, condition monitoring, and operation maintenance of screw rotors. Therefore, it is of significant importance to ensure high machining precision of screw rotors.

A pair of conjugate screw rotors is the most crucial component of the twin-screw compressor, whereby the machining precision of the screw rotors can greatly affect performance of the compressor. Screw rotors are treated as helical gears with nonparallel and nonintersecting or crossed axes. And the machining of helical gears can be applied to the machining of screw rotors. Screw rotors are machined today mainly by grinding or milling. Usually two stages are needed: the first is the roughing, where the workpiece is machined to its approximate size; the second is the finishing, where the rotors are machined to their final dimensions.

Due to a variety of internal and external factors of processing system of the machine tool, the machining errors seriously affected the precision and quality of rotor profile. 
There are two common technologies to improve the accuracy of rotor profile: direct compensation method and indirect compensation method. The machine tool is the compensation object for the indirect compensation method which improves the processing precision of rotor profiles by enhancing the precision of machine tool. Meanwhile, rotor products are the object for the direct compensation which makes direct error compensation to rotor profile. Indirect compensation method is realized by creating a new error artificially to offset the original one, which can decrease the machining error and improve the accuracy. However, this method has three shortcomings: lack of an ideal modeling, obvious technical defects in hardware error compensation, and immature software error compensation. For the direct compensation method, recent researches mainly focus on the change of rotor profile under different errors. There are many errors being discussed, including machine tool errors, parameter errors of rotor structure, and wear of forming tool. Therefore, the theory on direct compensation method of rotor profiles could be further investigated.

Stosic et al. [6] presented a method of general geometrical definitions of screw machine rotors and their manufacturing tools. Wu et al. [7] proposed a design method of forming wheel edge shape for screw rotors machining when arbitrary rotor profile is given. A contact line equation between the rotor tooth surface and the forming wheel revolving surface is derived based on the meshing theory, and then the calculation method for theoretical edge shape of forming wheel is proposed, which is applicable to both analytical and discrete forms of the rotor profile. These methods are extensively applied in this study to calculate forming tool profile and normal vector of each point of rotor profile.

Guo and Tang [8] presented a model-based simulation to analyze the influence of manufacturing process variations on rotor profile, this simulation serves as a virtual machine tool to machine rotors. By varying machine parameters within their tolerance bands, the simulation predicts rotor profile errors caused by various process parameter errors. Han and Meng [9] presented a new ZK worm mathematical model which involves several dressing parameters of cone grinding wheel, and then the effects of worm parameters on the normal tooth profile error along with the changing of the grinding wheel radius are analyzed. Stosic $[10,11]$ introduced three possible errors of forming tool set errors, namely, mismatch of the angle between the tool and rotor shafts, mismatch of the centre distance between the tool and the rotor, and mismatch in the axial tool position, to quantify the tool set errors as well as compensate the errors of rotor profile. $\mathrm{Wu}$ [12] also defined three error factors based on the coordinate system established, and then the effects of rotor profile error along with the changing of these three error factors are analyzed. These studies can only compensate the errors of rotor profile which distribute regularly. These methods focus on finding the changing law of rotor profile under different errors, and limited compensation case only fits the situation when errors distribute regularly, not suitable for random distribution.

In the study presented here, a novel method of error compensation is proposed to reduce the errors of rotor profile. The errors of rotor profile are obtained by trial machining of

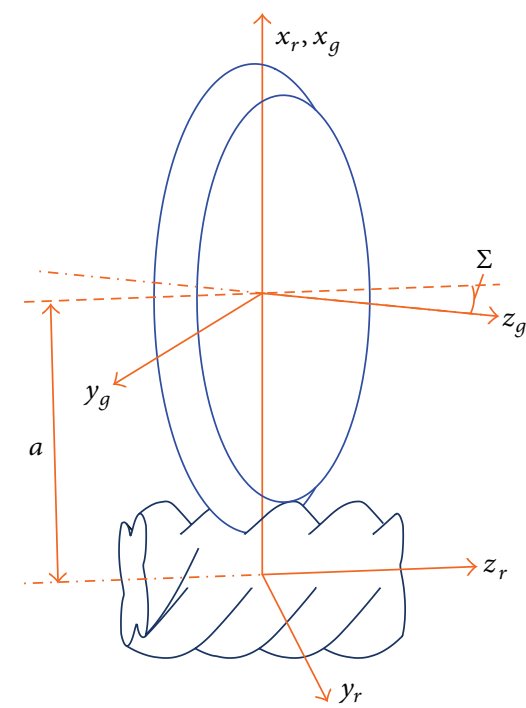

FIGURE 1: Coordinate system of forming tool for machining screw rotor.

rotors and then fitted into piecewise smooth data. A matrix of error compensation is established firstly, and then the errors of rotor profile are computed by this matrix to generate a compensated rotor profile. The compensated rotor profile is used to recalculate a new forming tool profile for machining rotor. The errors of new machined rotor profile are smaller than before and can be reduced within $(-0.01 \mathrm{~mm}, 0.01 \mathrm{~mm})$ after compensations. Finally, the actual machining examples illustrate the method of error compensation which can not only satisfy the machining requirement of high-precision rotors, but also has the characteristics of good stability and applicability.

\section{The Calculation of Forming Tool Profile}

As is shown in Figure 1, a coordinate system of the forming tool machining for screw rotor is presented. The coordinate system $S_{r}$ is attached to the rotor, rotating around the $z_{r}$-axis, while the coordinate system $S_{g}$ is affixed to the forming tool, rotating around the $z_{g}$-axis. The center distance $A$ is defined as the minimum distance between the $z_{r}$-axis and the $z_{g}$-axis, and $\Sigma$ is the shaft angle between two axes. The coordinate transformation from $S_{r}$ to $S_{g}$ can be derived as

$$
\left[\begin{array}{l}
x_{g} \\
y_{g} \\
z_{g}
\end{array}\right]=\left[\begin{array}{ccc}
1 & 0 & 0 \\
0 & \cos \Sigma & -\sin \Sigma \\
0 & \sin \Sigma & \cos \Sigma
\end{array}\right]\left[\begin{array}{l}
x_{r} \\
y_{r} \\
z_{r}
\end{array}\right]+\left[\begin{array}{c}
-a \\
0 \\
0
\end{array}\right]
$$

There is a contact line between the forming tool contour surface and the rotor helicoids during machining process. When the contact line spirally rotates around the $z_{r}$-axis, rotor helicoids are attained. And when the contact line rotates around $z_{g}$-axis, the contour surface of the forming tool would be shaped. The rotor profile consisted of discrete points, and the derivative of each discrete point of rotor profile is not given. To calculate the derivative, the method of accumulated chord length parameter, which can solve the problem of 
the large deflection of rotor profiles, is used in this paper. Assuming the coordinate of the discrete points is $P=$ $\left\{\left(x_{i}, y_{i}\right), i=1,2, \ldots, n\right\}$, the equation of rotor profile can be obtained as

$$
\mathbf{r}_{0}=[x(s), y(s)]
$$

where $s$ is the accumulated chord length parameter. And then the derivative of rotor profile can be attained as

$$
\mathbf{T}=\left[\frac{\partial x(s)}{\partial s}, \frac{\partial y(s)}{\partial s}\right]
$$

The helicoids of the rotors can be expressed by the following equation:

$$
\begin{aligned}
& \mathbf{r}(s, \theta) \\
& =[x, y, z] \\
& =[x(s) \cos \theta-e y(s) \sin \theta, e x(s) \sin \theta+y(s) \cos \theta, p \theta],
\end{aligned}
$$

where $e=1$ for the right-hand rotor, $e=-1$ for the lefthand rotor, $\theta$ is the rotation angle of the rotor, and $p=P z / 2 \pi$ is helix parameter, which means that the rotor translates a distance of lead $\mathrm{Pz}$ when it rotates one turn. The equation of helicoids of the rotor has been obtained, and the partial derivatives of the helicoids can be calculated as follows:

$$
\begin{gathered}
\mathbf{r}_{s}=\left[\frac{\partial x(s)}{\partial s} \cos \theta-e \frac{\partial y(s)}{\partial s} \sin \theta, e \frac{\partial x(s)}{\partial s} \sin \theta\right. \\
\left.+\frac{\partial y(s)}{\partial s} \cos \theta, 0\right], \\
\mathbf{r}_{\theta}=[-x(s) \sin \theta-e y(s) \cos \theta, e x(s) \cos \theta \\
-y(s) \sin \theta, p] .
\end{gathered}
$$

And then the normal vector of the helicoids of the rotor can be presented as

$$
\begin{aligned}
\mathbf{n}= & {\left[n_{x}, n_{y}, n_{z}\right] } \\
= & \left(\mathbf{r}_{s} \times \mathbf{r}_{\theta}\right)^{T} \\
= & {\left[p\left(e \frac{\partial x(s)}{\partial s} \sin \theta+\frac{\partial y(s)}{\partial s} \cos \theta\right),\right.} \\
& p\left(-\frac{\partial x(s)}{\partial s} \cos \theta+e \frac{\partial y(s)}{\partial s} \sin \theta\right), \\
& \left.e\left(x(s) \frac{\partial x(s)}{\partial s}+y(s) \frac{\partial y(s)}{\partial s}\right)\right] .
\end{aligned}
$$

According to envelope principle, the relative velocity $\mathbf{v}$ between the rotor and the forming tool can be presented as

$$
\begin{aligned}
\mathbf{v} & =\left[v_{x}, v_{y}, v_{z}\right] \\
& =[y \cos \Sigma+z \sin \Sigma,(x-a) \cos \Sigma,(x-a) \sin \Sigma] .
\end{aligned}
$$

In order to determine the relationship equation between the profile parameter $s$ and the rotational angle $\theta$, the enveloping condition [13] is that the common normal vector $\mathbf{n}$ at the contact point is perpendicular to the relative velocity $\mathbf{v}$ direction between two conjugate surfaces and can be shown as follows:

$$
\begin{aligned}
\mathbf{v} \cdot \mathbf{n}= & -p \cos \Sigma[e x(s) \sin \theta+y(s) \cos \theta] \\
& \times\left[e \frac{\partial x(s)}{\partial s} \sin \theta+\frac{\partial y(s)}{\partial s} \cos \theta\right] \\
& -p \cos \Sigma[x(s) \cos \theta-e y(s) \sin \theta-a] \\
& \times\left[\frac{\partial x(s)}{\partial s} \cos \theta-e \frac{\partial y(s)}{\partial s} \sin \theta\right] \\
& +e \sin \Sigma[x(s) \cos \theta-e y(s) \sin \theta-a] \\
& \times\left[x(s) \frac{\partial x(s)}{\partial s}+y(s) \frac{\partial y(s)}{\partial s}\right] \\
& -p^{2} \theta \sin \Sigma\left[e \frac{\partial x(s)}{\partial s} \sin \theta+\frac{\partial y(s)}{\partial s} \cos \theta\right]=0
\end{aligned}
$$

Substituting the value of $s$ into (6) yields the corresponding value $\theta=f(s)$, for the rotational angle. Substituting the value of $s$ and $\theta$ back into (4), the equation of contact line can be expressed as

$$
\begin{aligned}
\mathbf{r}_{c r}= & {\left[x_{c r}, y_{c r}, z_{c r}\right] } \\
= & {[x(s) \cos f(s)-e y(s) \sin f(s), e x(s) \sin f(s)} \\
& \quad+y(s) \cos f(s), p f(s)] .
\end{aligned}
$$

Then transforming the equation of contact line $\mathbf{r}_{c r}$ from rotor coordinate system $S_{r}$ to forming tool coordinate system $S_{g}$,

$$
\begin{aligned}
& \mathbf{r}_{c g}^{T} \\
& =\left[\begin{array}{c}
x(s) \cos f(s)-e y(s) \sin f(s)-a \\
{[\operatorname{ex}(s) \sin f(s)+y(s) \cos f(s)] \cos \Sigma-p f(s) \sin \Sigma} \\
{[\operatorname{ex}(s) \sin f(s)+y(s) \cos f(s)] \sin \Sigma+p f(s) \cos \Sigma}
\end{array}\right] .
\end{aligned}
$$

The contour surface of the forming tool can be generated by rotating the contact line around its rotating axis, and the equation of forming tool can be presented as

$$
\mathbf{r}_{g}=\left[Z_{g}, R_{g}\right]=\left[z_{c g}, \sqrt{x_{c g}^{2}+y_{c g}^{2}}\right]
$$

\section{The Error Compensation Method of Rotor Profile}

3.1. Analysis of Error Source. The errors of rotor profile are mainly caused by the comprehensive errors of machine tool which can be divided into two major categories of external and internal errors according to its own attributes, as is shown in Figure 2. The internal error is composed of geometric 


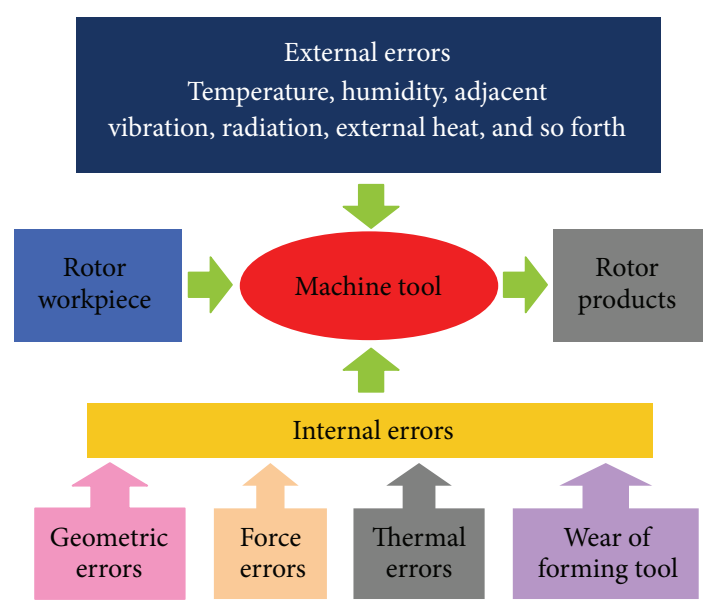

FIGURE 2: Classification of errors.

TABLE 1: The proportion of internal error factors.

\begin{tabular}{lc}
\hline Factors & Proportion \\
\hline Geometric errors & $35 \%$ \\
Thermal errors & $18 \%$ \\
Force errors & $12 \%$ \\
Wear of forming tool & $10 \%$ \\
Deformation error of workpiece & $8 \%$ \\
Setting error & $3 \%$ \\
Fixture error & $5 \%$ \\
Others & $9 \%$ \\
\hline
\end{tabular}

errors, force errors, thermal errors, wear of forming tool, and its own vibration. The external error mainly considers the influence of external environment including temperature, humidity, and adjacent vibration. Ignoring the external error, according to "machine tool, fixture, rotor, and forming tool" machining system, the internal error can be divided into more detailed categories, and the distribution of each error factor is roughly shown in Table 1.

3.2. Method of Error Compensation. To improve the machining precision of rotor profile, this study presents a novel compensation method for rotor profile errors. In order to compare with the errors between different rotor profiles, the overall error of rotor profile $\eta$ is introduced as an evaluation index:

$$
\eta=\frac{\left(\sum_{i=1}^{n} \delta_{i}^{2}\right)^{1 / 2}}{n},
$$

where $\delta_{i}$ is the error of each point of rotor profile.

As is shown in Figure 3, the compensation procedure can be divided into the following steps.

(1) According to the theoretical rotor profile, the profile of forming tool is first calculated. On the basis of this profile, a forming tool is dressed and then used to process a rotor workpiece to obtain a rotor product.
Errors of rotor profile are obtained by measuring the machined rotor, and the overall error $\eta_{1}$ can be calculated.

(2) The measured error of rotor profile needs to be processed on account of its large fluctuation feature. According to the trend of error curve $a b, b c, c d$, de, ef, $f g, g h, h i$, and $i j$, errors are divided into 9 sections. If the radius of probe is larger than that of curvature at the root of the rotor, the points are unable to be measured; see ef section. A linear transition can be used to fit the section $e f$. So the fitting equation of $a b$, $b c, c d, d e, e f, f g, g h, h i$, and $i j$ can be expressed as follows:

$$
\delta_{i}= \begin{cases}0.00166 i+0.00387, & i \in[1,10], \\ -0.0016 i+0.03639, & i \in(10,25], \\ -0.000065 i+0.00706, & i \in(25,106], \\ 0.00296 i-0.31445, & i \in(106,117], \\ -0.001 i+0.148, & i \in(117,147], \\ 0, & i \in(147,169], \\ 0.01 i-1.69, & i \in(169,174], \\ -0.00286 i+0.56302, & i \in(174,212], \\ 0.00115 i-0.2879, & i \in(212,325] \\ -0.01091 i+3.63172, & i \in(325,335] \\ 0.000366 i-0.13513, & i \in(335,340]\end{cases}
$$

(3) The errors data has been reconstructed, and then the matrix of error compensation is established to generate a compensated rotor profile:

$$
\begin{aligned}
\mathbf{r}_{s} & =\left[\begin{array}{cc}
x_{s 1} & y_{s 1} \\
x_{s 2} & y_{s 2} \\
\vdots & \vdots \\
x_{s i} & y_{s i} \\
\vdots & \vdots \\
x_{s n-1} & y_{s n-1} \\
x_{s n} & y_{s n} \\
1 & 0 \\
0 & 1
\end{array}\right] \\
& =\left[\begin{array}{ccccccccc}
\delta_{1} & 0 & 0 & 0 & 0 & 0 & 0 & x_{1} & y_{1} \\
0 & \delta_{2} & 0 & 0 & 0 & 0 & 0 & x_{2} & y_{2} \\
\vdots & \vdots & \vdots & \vdots & \vdots & \vdots & \vdots & \vdots & \vdots \\
0 & 0 & 0 & \delta_{i} & 0 & 0 & 0 & x_{i} & y_{i} \\
\vdots & \vdots & \vdots & \vdots & \vdots & \vdots & \vdots & \vdots & \vdots \\
0 & 0 & 0 & 0 & 0 & \delta_{n-1} & 0 & x_{n-1} & y_{n-1} \\
0 & 0 & 0 & 0 & 0 & 0 & \delta_{n} & x_{n} & y_{n} \\
0 & 0 & 0 & 0 & 0 & 0 & 0 & 1 & 0 \\
0 & 0 & 0 & 0 & 0 & 0 & 0 & 0 & 1
\end{array}\right]
\end{aligned}
$$




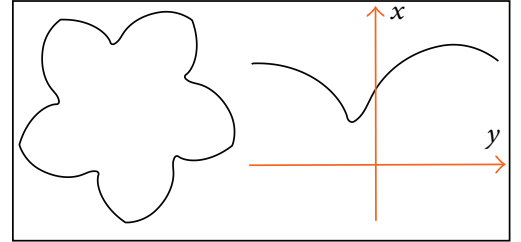

Theoretical rotor profile

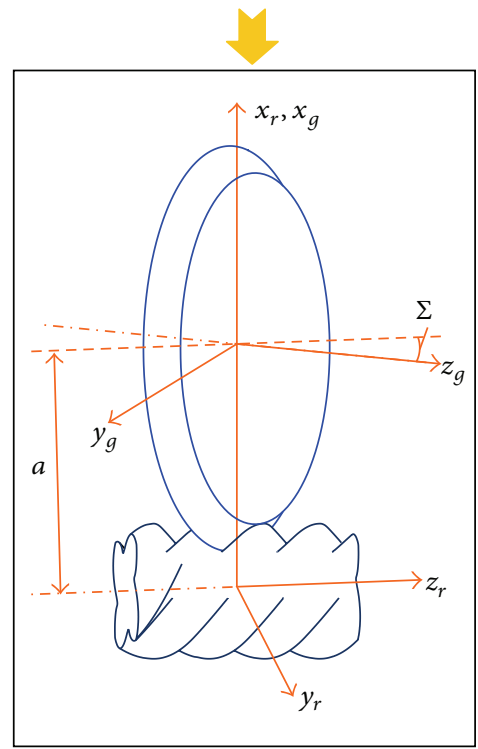

Rotors machining

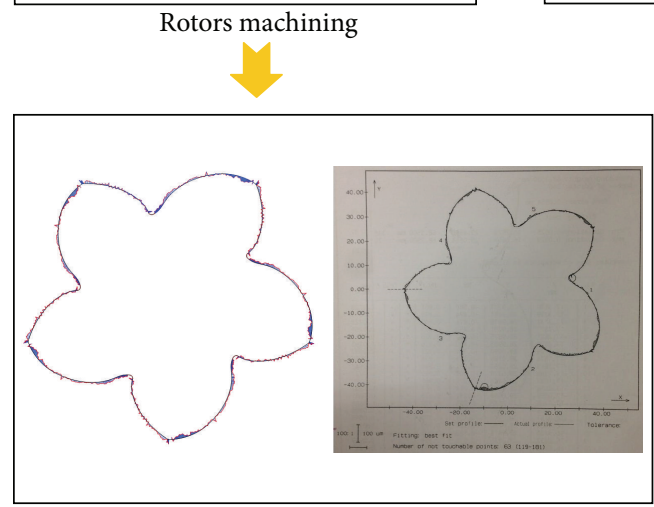

Rotor products
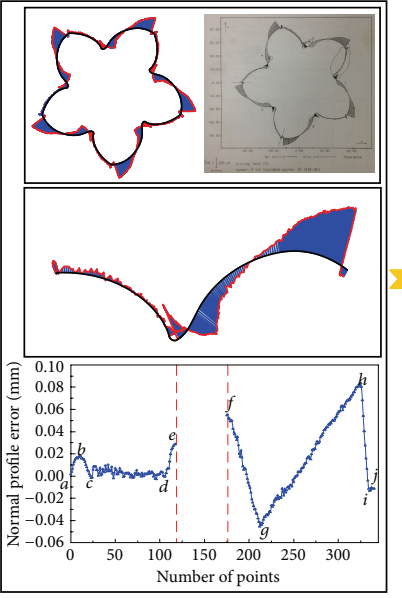

Errors of rotor profile

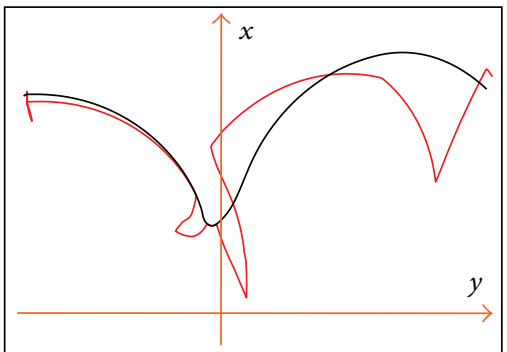

Compensated rotor profile

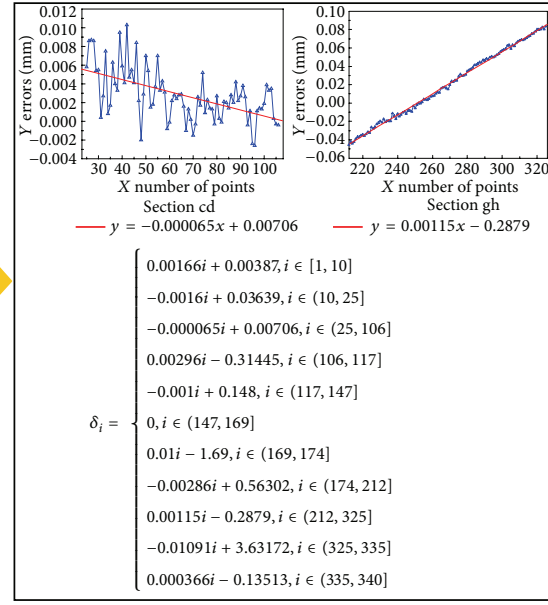

Fitting of error data
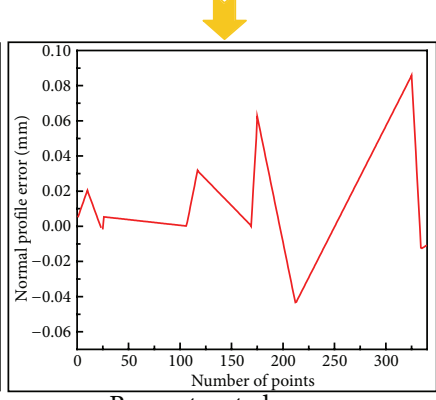

Reconstructed errors

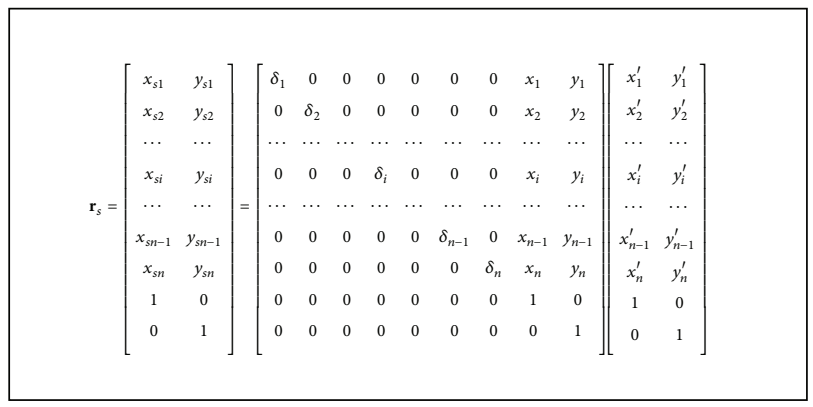

Matrix of error compensation

FIGURE 3: Procedure of error compensation.

$\times\left[\begin{array}{cc}x_{1}^{\prime} & y_{1}^{\prime} \\ x_{2}^{\prime} & y_{2}^{\prime} \\ \vdots & \vdots \\ x_{i}^{\prime} & y_{i}^{\prime} \\ \vdots & \vdots \\ x_{n-1}^{\prime} & y_{n-1}^{\prime} \\ x_{n}^{\prime} & y_{n}^{\prime} \\ 1 & 0 \\ 0 & 1\end{array}\right]$,

where $\mathbf{r}_{s}=\left(x_{s i}, y_{s i}\right)$ is the compensated rotor profile and $\mathbf{r}_{0}=\left(x_{i}, y_{i}\right)$ is the theoretical rotor profile. $\mathbf{n}_{0}=$ $\left(x_{i}^{\prime}, y_{i}^{\prime}\right)$ is the normal vector of theoretical rotor profile and can be calculated as follows:

$$
\begin{aligned}
\mathbf{n}_{0} & =\left(x_{i}^{\prime}, y_{i}^{\prime}\right) \\
& =\left[\frac{\partial y_{i} / \partial s}{\sqrt{\left(\partial x_{i} / \partial s\right)^{2}+\left(\partial y_{i} / \partial s\right)^{2}}}, \frac{-\partial x_{i} / \partial s}{\sqrt{\left(\partial x_{i} / \partial s\right)^{2}+\left(\partial y_{i} / \partial s\right)^{2}}}\right] .
\end{aligned}
$$

(4) Finally, the rotor profile $\mathbf{r}_{s}$ can be used to recalculate the forming tool profile by (2)-(11). After a new rotor is machined, the overall error of new rotor profile $\eta_{2}$ is calculated. By comparing the values of $\eta_{1}$ and $\eta_{2}$, the errors after compensation are smaller than the errors 


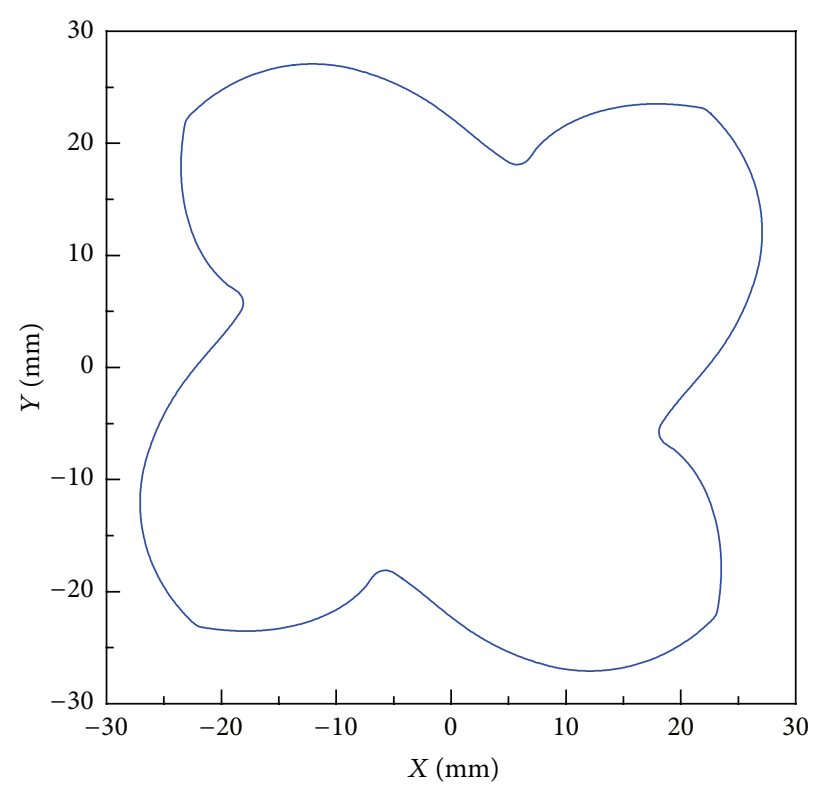

(a) Male rotor

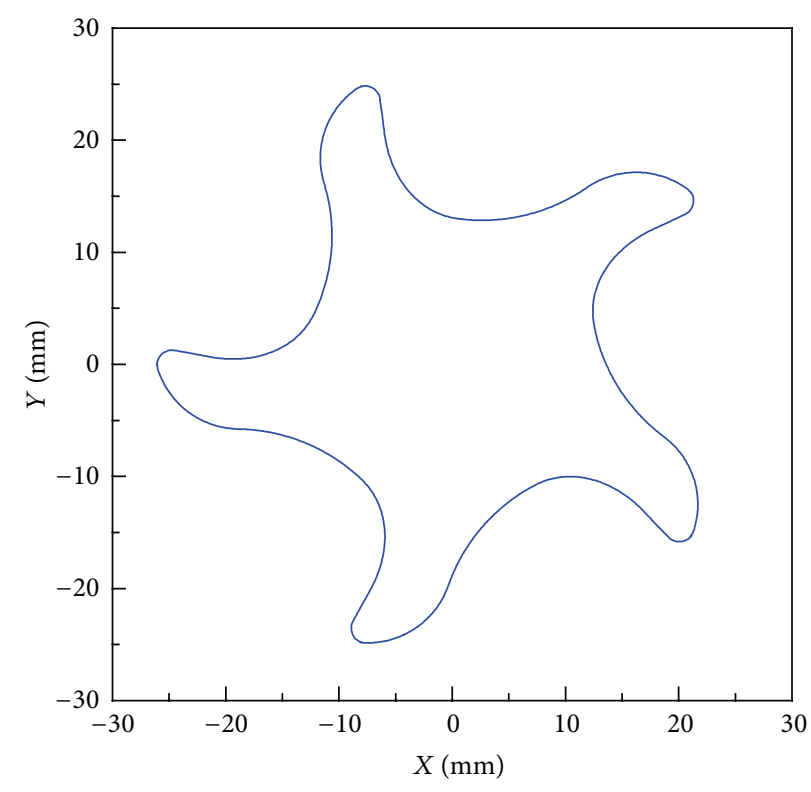

(b) Female rotor

Figure 4: Cross-section contour of rotors.

before compensation, and that can be reduced within $(-0.01 \mathrm{~mm}, 0.01 \mathrm{~mm})$. Thus, the error compensation of rotor profile is achieved.

\section{Example}

The method above has been successfully applied to many screw compressor manufacturers. Taking a machining example of the male rotor and female rotor which were supplied from a screw compressor manufacturer, the parameters of the rotor are given in Table 2 . Figure 4 shows the crosssection contour of the male rotor and female rotor; they are composed of discrete points. The forming tool profiles of male and female rotors are calculated by (2)-(11) and shown in Figure 5.

Figure 6 shows the process of machining rotors, and the machined rotor products are shown in Figure 7. To assess the precision of rotor profile, the end face of rotor is measured by 3D CMM, as is shown in Figure 8.

The measurement results of male and female rotors profile are shown in Figures 9 and 10, in which the errors are magnified by 50 times, and the tolerance of rotor profile is $(-0.01 \mathrm{~mm}, 0.01 \mathrm{~mm})$. Figure 9 shows the rotor profiles after trial machining, of which the overall errors are $\eta_{\text {male_1 }}=$ $0.06675 \mathrm{~mm}$ and $\eta_{\text {female_1 }}=0.1839 \mathrm{~mm}$ under the effect of comprehensive errors of machine tool. The method of error compensation is used to reduce the errors as is shown in Figure 10; the overall errors of male rotor and female rotor are $\eta_{\text {male_2 }}=0.00785 \mathrm{~mm}$ and $\eta_{\text {female_2 }}=0.0127 \mathrm{~mm}$. By comparing the value of $\eta_{\text {male_1 }}$ and $\eta_{\text {male_2 }}$ and $\eta_{\text {female_1 }}$ and $\eta_{\text {female } 2}$, the errors after compensation are far less than the errors before compensation. Thus, the method of error compensation can satisfy the machining requirement of high-precision rotors.

In the mass production of screw rotors, the precision of rotor profiles is tracked. Figure 11 shows 20 consecutive machined products of the same rotor profile, and the overall errors distribute within $(0.005 \mathrm{~mm},-0.0094 \mathrm{~mm})$. Figure 12 shows the comparison of profile errors of 5 different rotor products before and after compensation. The result shows that, after compensation, all errors of 5 different rotor products are reduced. Figures 11 and 12 illustrate that the method of error compensation is stable and applicable.

\section{Conclusions}

(1) This paper investigates the envelope condition to derive an equation of the contact line, and then the contact line is used to generate the forming tool profile.

(2) After analyzing the error sources of machine tool, a method of error compensation is presented to improve the accuracy of rotor profile. The errors of rotor profile are obtained by small sample machining trials of rotors and then fitted into piecewise smooth data. A matrix of error compensation is established firstly, and then the errors of rotor profile are computed by this matrix to generate a compensated rotor profile. The compensated rotor profile is then used to regenerate forming tool and a new rotor product is processed on the same machine tool. And the errors of new rotor profile are smaller and can be reduced within $(-0.01 \mathrm{~mm}, 0.01 \mathrm{~mm})$ after compensations. 


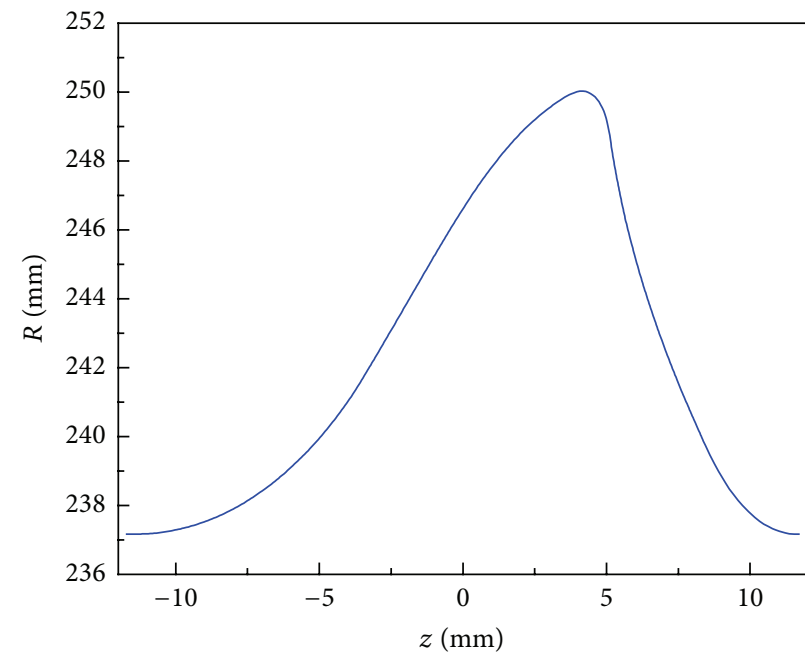

(a) Male rotor

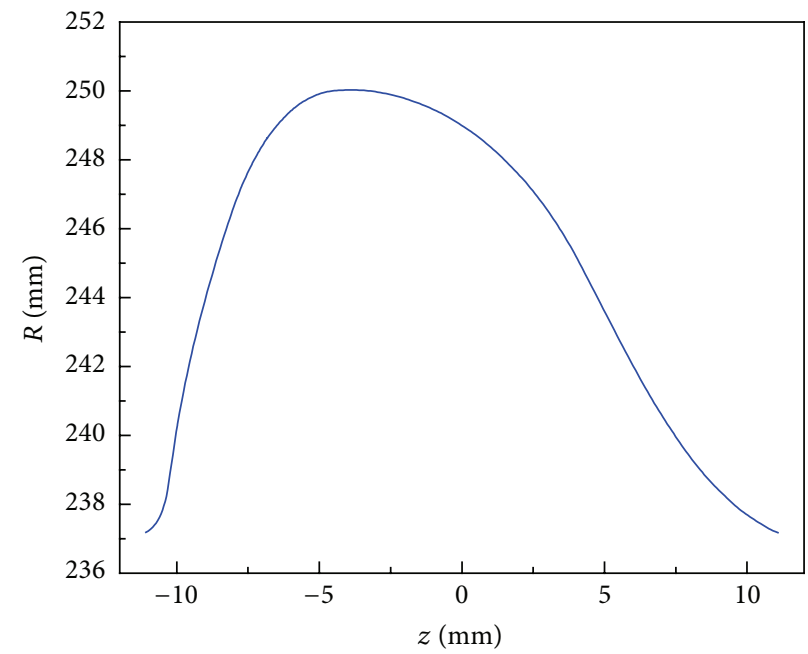

(b) Female rotor

FIGURE 5: Forming tool profile of rotors.

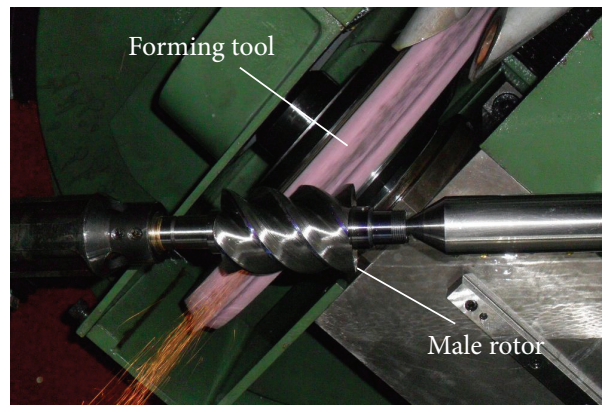

(a)

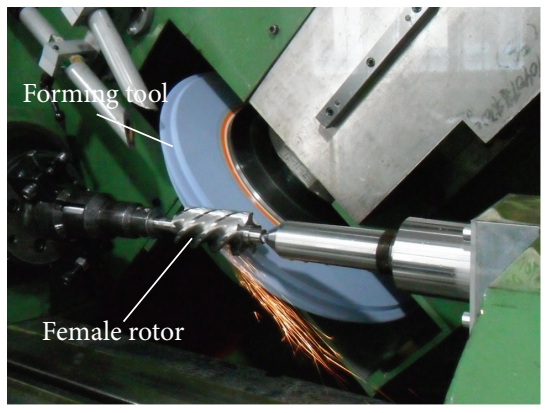

(b)

Figure 6: The machining of rotors.

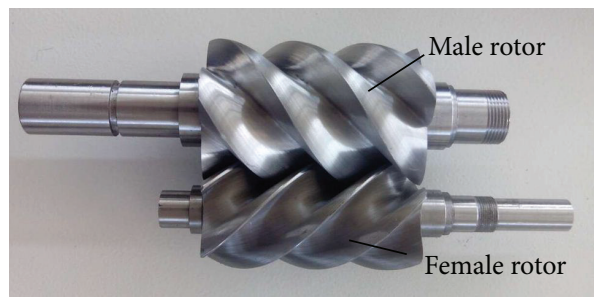

FIGURE 7: Rotor products.

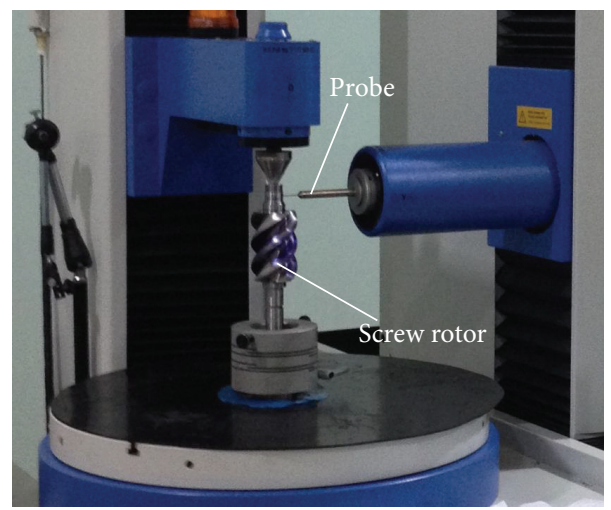

Figure 8: Measurement of rotors. 


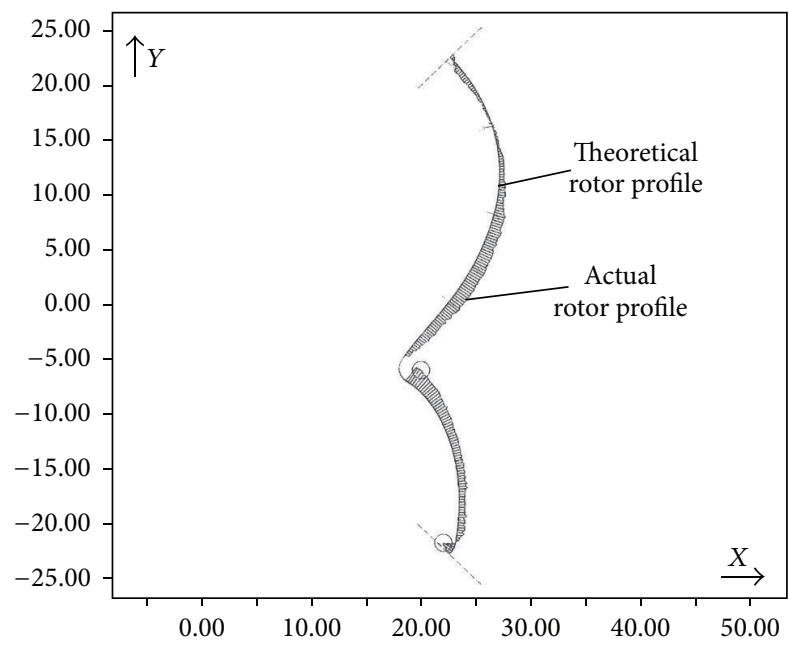

(a) Male rotor

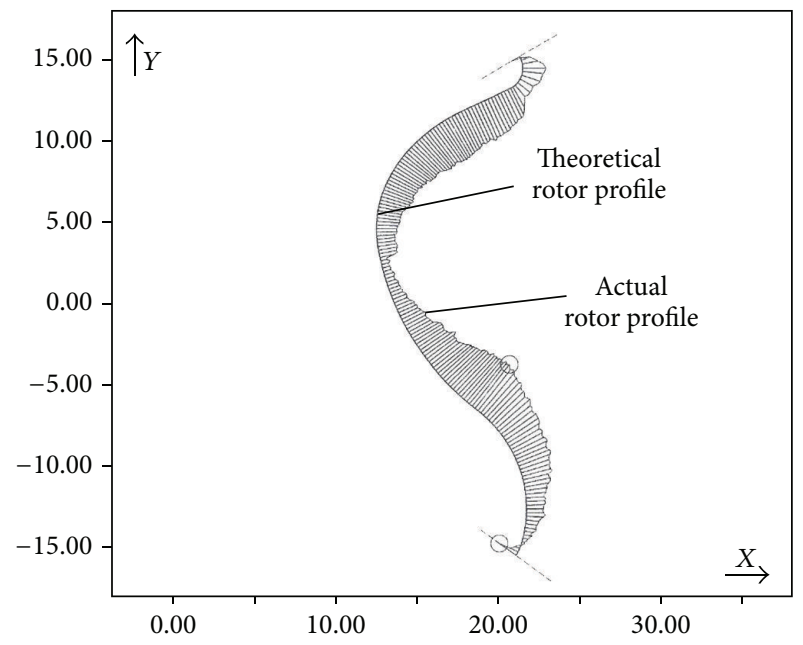

(b) Female rotor

FIGURE 9: Rotor profile before compensation.

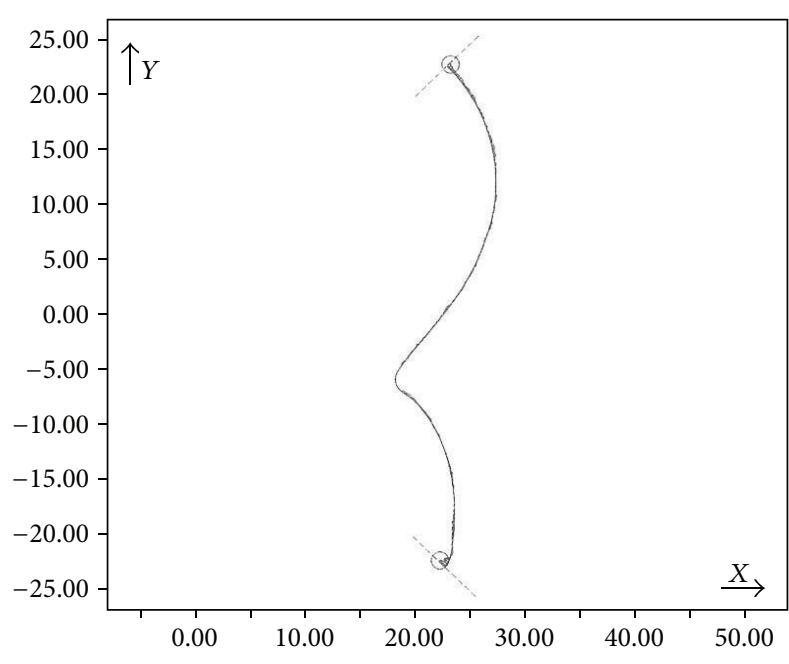

(a) Male rotor

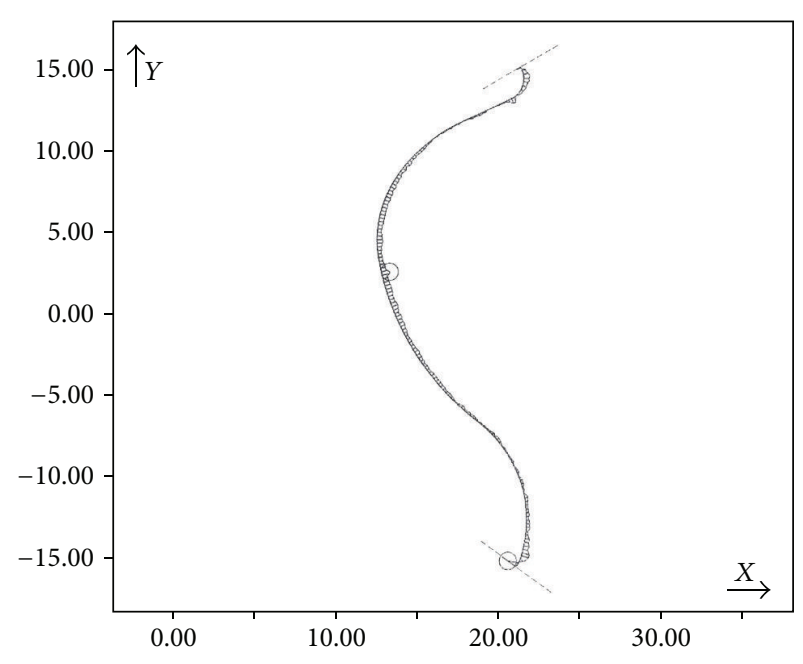

(b) Female rotor

Figure 10: Rotor profile after compensation.

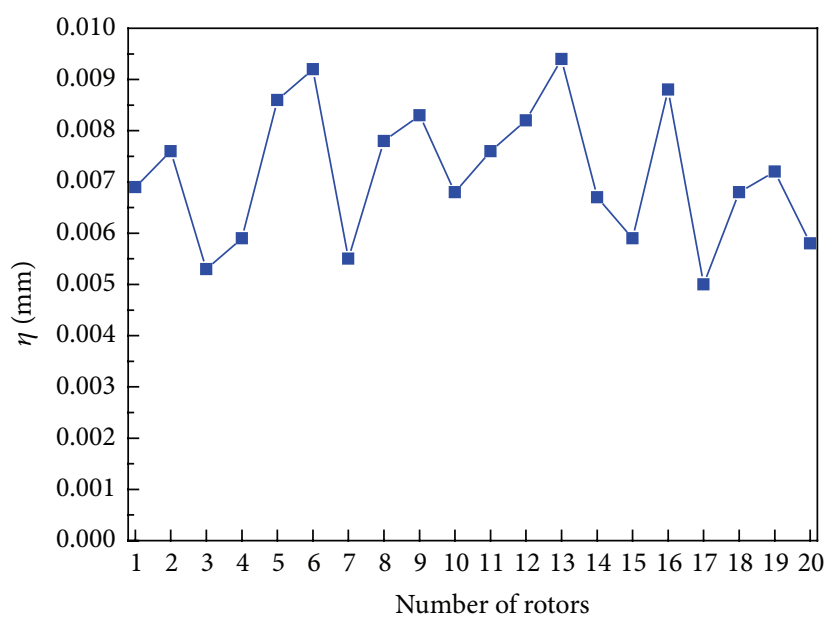

FIGURE 11: Overall errors of 20 consecutive machining rotor products. 
TABLE 2: The parameters of rotor.

\begin{tabular}{|c|c|c|c|c|c|c|c|}
\hline Type & Revolving direction & Number of teeth & Outer diameter $(\mathrm{mm})$ & Root diameter (mm) & Lead $(\mathrm{mm})$ & Helical angle (deg) & Material \\
\hline Male & Right-hand & 4 & 68.25 & 39.7635 & 128 & \multirow{2}{*}{45} & \multirow{2}{*}{ 42CrMo } \\
\hline Female & Left-hand & 5 & 54.653 & 27.248 & 160 & & \\
\hline
\end{tabular}

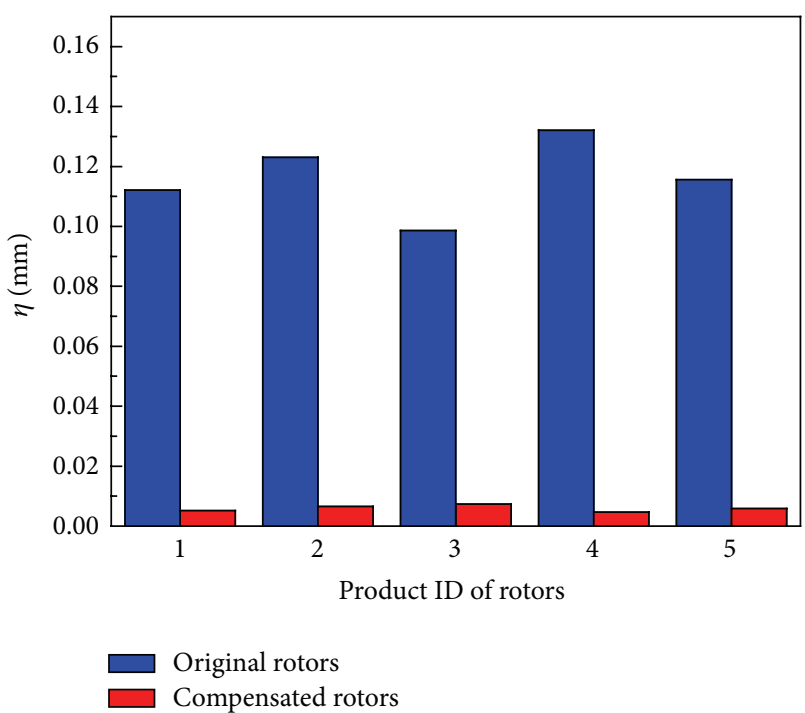

FIGURE 12: Comparison of profile errors of 5 different rotor products.

(3) Finally, the actual five machining examples in the high precision, stability, and applicability illustrate that the method of error compensation can not only satisfy the machining requirements of high-precision rotors, but also has the characteristics of good stability and applicability.

\section{Conflict of Interests}

The authors declare that there is no conflict of interests regarding the publication of this paper.

\section{Acknowledgment}

This work was financially supported by the Science and Technology Innovation Project of Shannxi Province under Grant no. 2014KTDZ01-04.

\section{References}

[1] Y. Lei, J. Lin, Z. He, and Y. Zi, "Application of an improved kurtogram method for fault diagnosis of rolling element bearings," Mechanical Systems and Signal Processing, vol. 25, no. 5, pp. 1738-1749, 2011.

[2] Y. Lei, M. J. Zuo, Z. He, and Y. Zi, "A multidimensional hybrid intelligent method for gear fault diagnosis," Expert Systems with Applications, vol. 37, no. 2, pp. 1419-1430, 2010.

[3] Y. Wang and M. Liang, "Identification of multiple transient faults based on the adaptive spectral kurtosis method," Journal of Sound and Vibration, vol. 331, no. 2, pp. 470-486, 2012.
[4] J. Xiang, T. Matsumoto, J. Long, Y. Wang, and Z. Jiang, "A simple method to detect cracks in beam-like structures," Smart Structures and Systems, vol. 9, no. 4, pp. 335-353, 2012.

[5] J. Xiang, T. Matsumoto, Y. Wang, and Z. Jiang, "A hybrid of interval wavelets and wavelet finite element model for damage detection in structures," CMES: Computer Modeling in Engineering \& Sciences, vol. 81, no. 3-4, pp. 269-294, 2011.

[6] N. Stosic, I. K. Smith, A. Kovacevic, and E. Mujic, "Geometry of screw compressor rotors and their tools," Journal of Zhejiang University: Science A, vol. 12, no. 4, pp. 310-326, 2011.

[7] B. Wu, J. Zhang, J. Yang, and D. Zhang, "Calculation method for edge shape of forming wheel for screw rotors machining," Journal of Mechanical Engineering, vol. 48, no. 19, pp. 192-198, 2012 (Chinese).

[8] C. Guo and Y. Tang, "Influence of process parameters on screw rotor profiles," Machining Science and Technology, vol. 7, no. 1, pp. 105-118, 2003.

[9] Y. P. Han and J. F. Meng, "Intellectualized dressing of the grinding wheel in the process of grinding ZK worm," Chinese Journal of Mechanical Engineering, vol. 40, no. 5, pp. 178-183, 2004 (Chinese).

[10] N. Stosic, "Evaluating errors in screw rotor machining by tool to rotor transformation," Proceedings of the Institution of Mechanical Engineers Part B: Journal of Engineering Manufacture, vol. 220, no. 10, pp. 1589-1596, 2006.

[11] N. Stosic, "A geometric approach to calculating tool wear in screw rotor machining," International Journal of Machine Tools and Manufacture, vol. 46, no. 15, pp. 1961-1965, 2006.

[12] R. Wu Y, Novel Design Methods of Rotor and Cutter Profiles for the Twin-Screw Compressor, National Chung-Cheng University, Chia-Yi, Taiwan, 2007.

[13] F. L. Litvin and A. Fuentes, Gear Geometry and Applied Theory, Cambridge University Press, Cambridge, Mass, USA, 2004. 

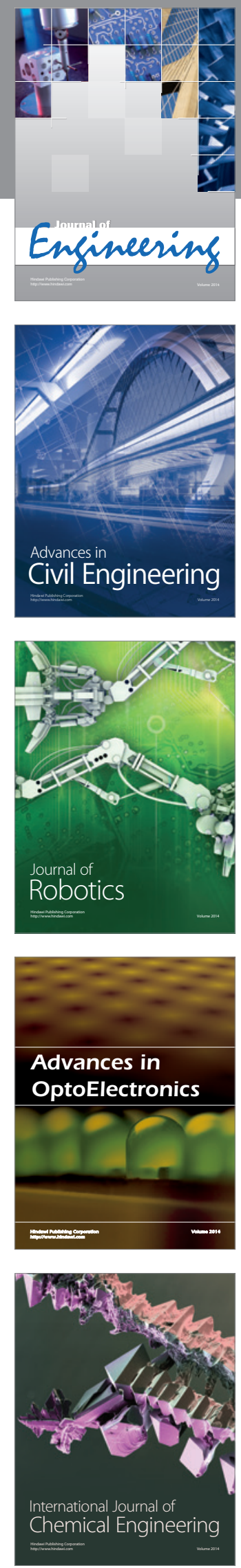

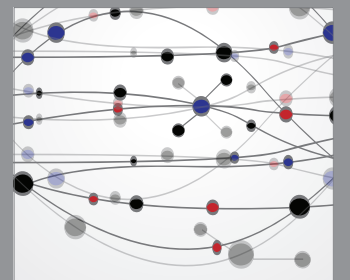

The Scientific World Journal
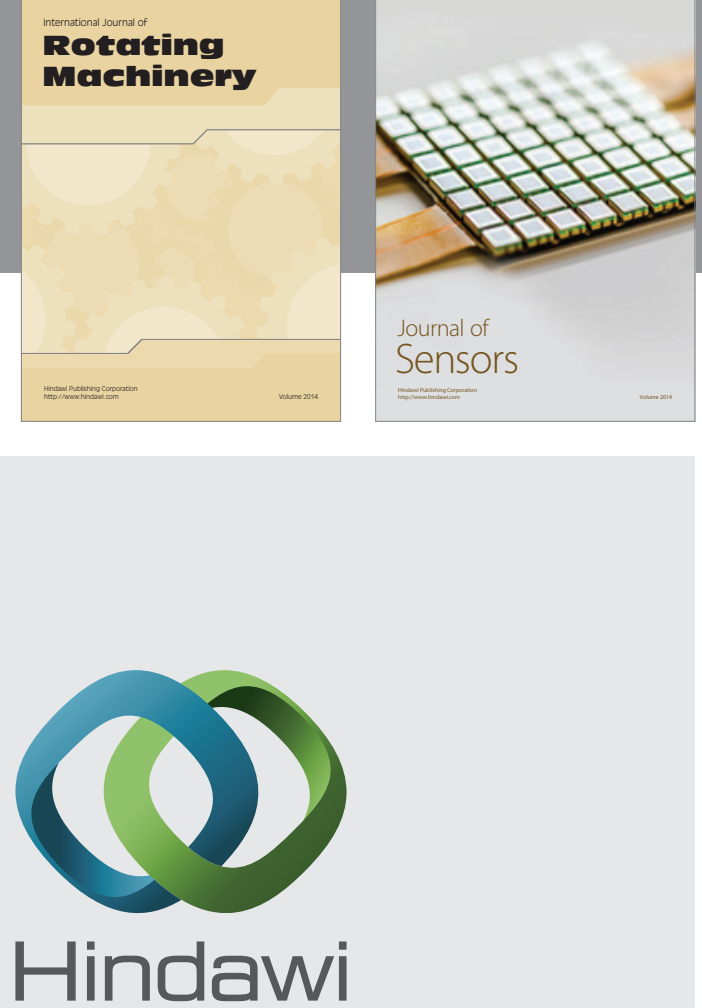

Submit your manuscripts at http://www.hindawi.com
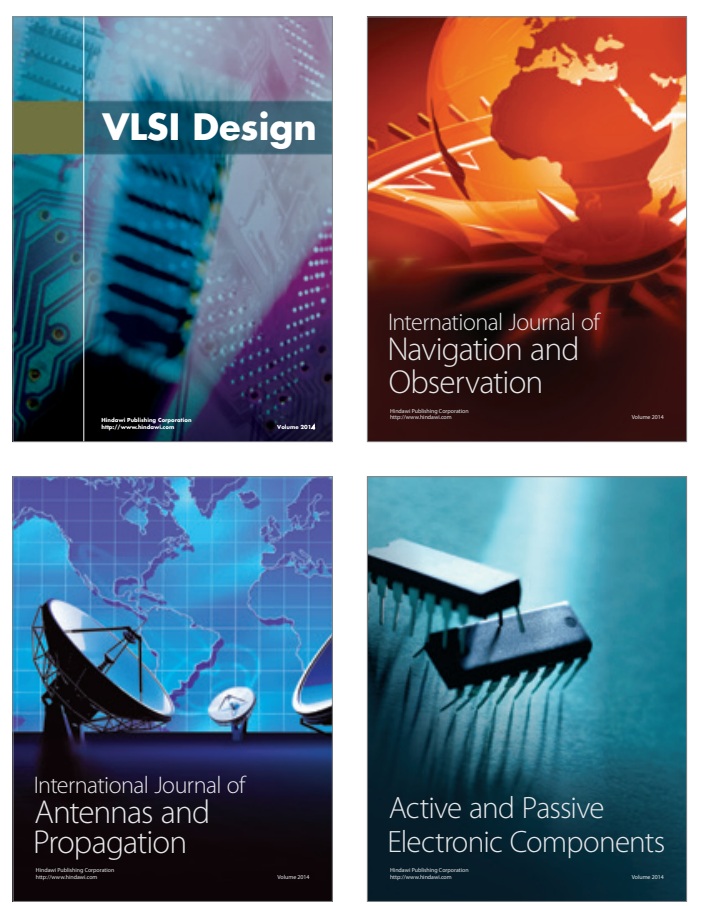
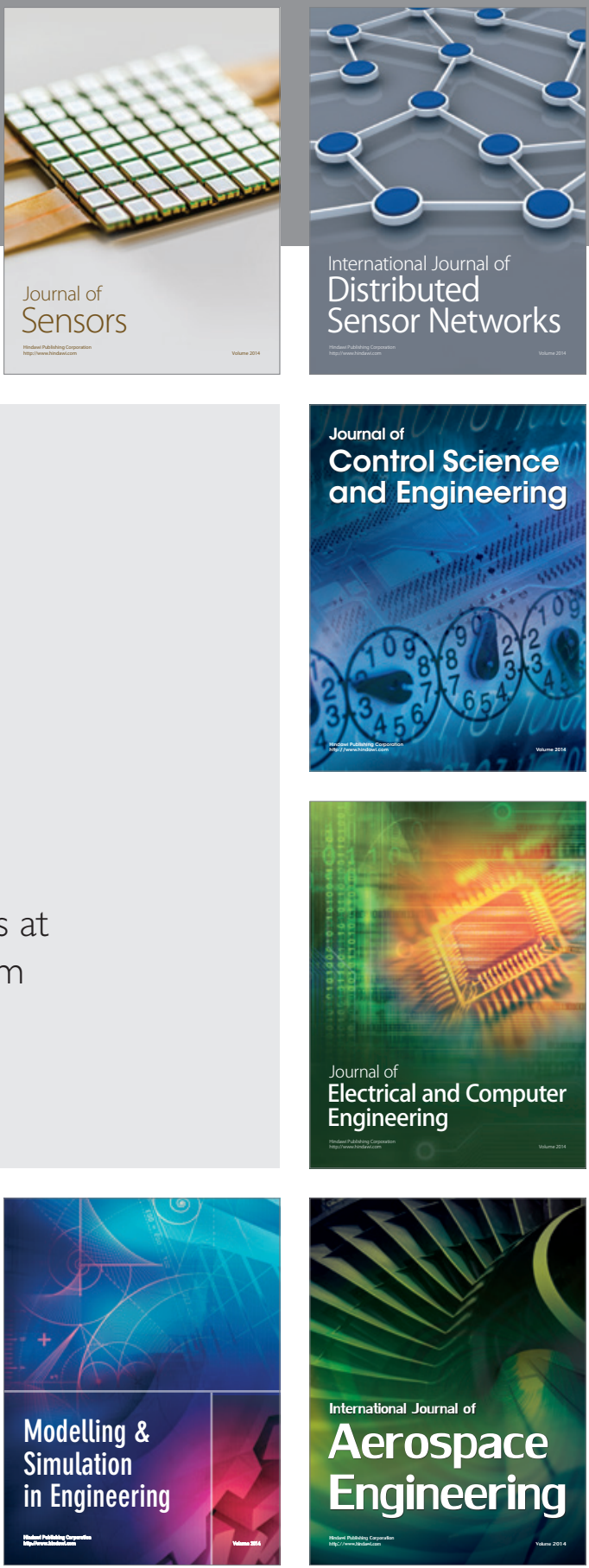

Journal of

Control Science

and Engineering
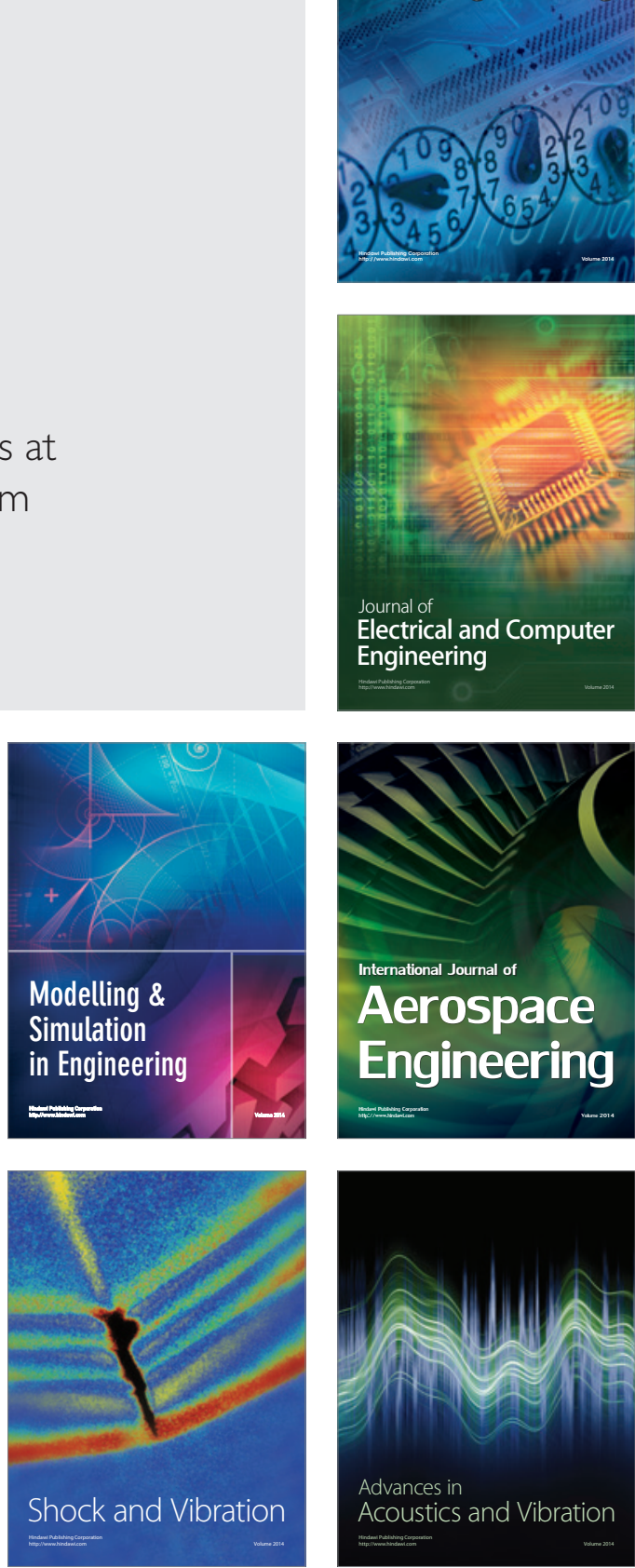